\title{
Philosophiques
}

\section{L’intentionnalité comme phénomène linguistique}

\section{Claude Panaccio}

Volume 8, numéro 2, octobre 1981

URI : https://id.erudit.org/iderudit/203168ar

DOI : https://doi.org/10.7202/203168ar

Aller au sommaire du numéro

Éditeur(s)

Société de philosophie du Québec

ISSN

0316-2923 (imprimé)

1492-1391 (numérique)

Découvrir la revue

Citer cet article

Panaccio, C. (1981). L'intentionnalité comme phénomène linguistique. Philosophiques, 8(2), 239-257. https://doi.org/10.7202/203168ar

\section{Résumé de l'article}

La notion phénoménologique d'intentionnalité suscite certaines énigmes philosophiques assez déroutantes concernant par exemple l'existence d'entités relationnelles (la conscience) ou le statut des objets intentionnels. Il est ici suggéré que ces énigmes, apparemment ontologiques, auraient plus de chances d'être élucidées si elles étaient considérées comme des problèmes sémantiques concernant cette catégorie spéciale d'énoncés que l'on appelle énoncés intensionnels ». Elles pourraient alors être discutées à l'aide de méthodes plus précises comme celles de Carnap, Church ou Quine. 


\title{
L'INTENTIONNALITÉ COMME PHÉNOMÈNE LINGUISTIQUE*
}

\author{
par Claude Panaccio
}

RÉSUMÉ. La notion phénoménologique d'intentionnalité suscite certaines énigmes philosophiques assez déroutantes concernant par exemple l'existence d'entités relationnelles (la conscience) ou le statut des objets intentionnels. Il est ici suggéré que ces énigmes, apparemment ontologiques, auraient plus de chances d'être élucidées si elles étaient considérées comme des problèmes sémantiques concernant cette catégorie spéciale d'énoncés que l'on appelle «énoncés intensionnels». Elles pourraient alors être discutées à l'aide de méthodes plus précises comme celles de Carnap, Church ou Quine.

ABSTRACT. The phenomenological notion of intentionality raises a number of rather perplexing philosophical riddles, for instance about the existence of relational entities (consciousness) or about the status of intentional objects. The paper suggests that these seemingly ontological riddles would gain more chances to be elucidated if they were regarded as semantic problems about a special kind of statements called «intensional statements». Thus they could be discussed with the help of some more precise methods such as those of Carnap, Church or Quine

«L'intentionnalité $(.$.$) , cette propriété remarquable à laquelle$ renvoient toutes les énigmes de la théorie de la raison et de la métaphysique». (Husserl, 1913; trad. franc., p. 283).

Le principe central de la phénoménologie - le principe d'intentionnalité - pose, on le sait, que toute conscience est conscience de quelque chose. Voilà qui suscite aussitôt certaines énigmes particulièrement délicates: pouvons-nous admettre l'existence empirique d'êtres intrinsèquement et essentiellement relationnels? Et si la conscience dont il est ici question n'a pas véritablement d'empiricité - s'il s'agit par exemple de la

*Une première version de ce texte a été présentée oralement au Congrès de l'Acfas, à Québec, mai 1980, dans le cadre d'une table ronde sur L'intentionnalité: phénoménologie versus positivisme et philosopbie analytique. Je remercie tout particulièrement Robert Nadeau et Claude-Elizabeth Perreault pour leurs commentaires encourageants et utiles. 
conscience «transcendantale» - , quel est au juste son rapport avec les êtres conscients que nous rencontrons à tout moment dans l'expérience familière, notamment les êtres humains? D'autre part, si l'intentionnalité est bien une relation, quels en sont les relata? Quel est en particulier le statut des objets intentionnels, ces entités étranges que Husserl appelle les «noèmes»? S'ils sont distincts des objets réels et s'ils ne se confondent pas avec des états psychologiques individuels, où faut-il les situer? Et comment reliera-t-on l'arbre noématique à l'arbre réel?

L'hypothèse que je voudrais explorer ici, de façon plus suggestive que systématique, est la suivante: ces énigmes phénoménologiques auraient plus de chances d'être élucidées si elles étaient traduites en problèmes logico-linguistiques et abordées à l'aide des instruments de l'analyse sémantique.

Je partirai de deux citations révélatrices, l'une de Lyotard (1961) et l'autre de Blondel (1934), pour tenter de montrer, à titre d'illustration, comment se dissipe leur apparente étrangeté lorsqu'elles sont interprétées comme des énoncés métalinguistiques. Puis j'examinerai, à larges traits, la façon dont pourraient être reformulées en termes logico-sémantiques certaines des principales énigmes de l'intentionnalité.

\section{DU PRINCIPE D'INTENTIONNALITÉ AU PRINCIPE DE PATERNITÉ}

Le passage de Lyotard qui me servira d'amorce à la discussion constitue justement une formulation, parmi d'autres possibles, du fameux principe d'intentionnalité:

Ma conscience ne peut pas être pensée si imaginairement on lui retire ce dont elle est conscience, on ne peut même pas dire qu'elle serait alors conscience de néant, puisque ce néant serait du même coup le phénomène dont elle serait conscience; la variation imaginaire opérée sur la conscience nous révèle bien ainsi son être propre qui est d'être conscience de quelque chose.

(Lyotard, 1961, p. 30).

Ce principe, dont on a rappelé plus haut la version abrégée ("toute conscience est conscience de quelque chose»), paraît bien requérir l'existence d'entités individuelles intrinsèquement relationnelles ("ma conscience»).

Introduisons maintenant, pour fins de comparaison, un autre principe, que j'appellerai le principe de paternité, susceptible d'une formulation rigoureusement parallèle à celle de Lyotard: 
Un père ne peut pas être pensé si imaginairement on lui retire ce dont il est le père, on ne peut même pas dire qu'il serait alors père de rien, puisque ce rien serait du même coup le phénomène dont il serait père; la variation imaginaire opérée sur le père nous révèle bien ainsi son être propre qui est d'être père de quelque chose.

Il existe aussi une version abrégée du principe de paternité:

tout père est père de quelque chose.

La formulation longue du principe de paternité, on le voit facilement, n'est qu'une manière tarabiscotée de dire que la paternité est une relation ${ }^{1}$, ce qui est vrai par définition: le principe de paternité est un principe analytique. Dire qu'il nous révèle l'être propre du père revient ici à affirmer qu'il nous révèle le sens - ou peut-être même la syntaxe — du mot «père». Le principe analytique de paternité pourrait être reformulé comme un postulat de signification (au sens de Carnap, 1952) qui poserait par définition que:

pour tout $x$, si $x$ est un père, alors il existe un $y$ tel que $x$ est le père de $y$.

Ce postulat de signification n'introduit dans l'ontologie aucune nouvelle entité mystérieuse et intrinsèquement relationnelle: les variables $\mathrm{x}$ et $\mathrm{y}$ continuent de prendre leurs valeurs dans l'univers des individus familiers.

Le principe d'intentionnalité peut recevoir le même traitement et apparaître comme un postulat de signification relatif au prédicat philosophique "être une conscience». Il se formulerait alors de la façon suivante (ou à peu près):

pour $\mathrm{x}$, si $\mathrm{x}$ est une conscience, alors il existe un $\mathrm{y}$ tel que $\mathrm{x}$ a conscience de $y$.

Dire que cela révèle l'être de la conscience, c'est en l'occurrence une manière trompeuse de dire que ce principe révèle (partiellement) le sens (ou la syntaxe) du mot "conscience», ou encore qu'il est analytique ${ }^{2}$. La fameuse variation imaginaire de la phénoménologie n'est ici rien d'autre qu'un test d'analyticité.

1. C'est là une formulation un peu lâche. Ceux qui aiment l'exactitude auront remarqué que notre principe de paternité affirme en fait ceci: le prédicat unaire «être père» n'est satisfait que par les individus qui appartiennent au domaine de la relation «être père de». Mais cette complication n'est pas ici pertinente.

2. L'analyticité du principe ne tient évidemment pas à la forme logique de l'énoncé, mais exclusivement au fait qu'il s'agit d'un postulat de signification. 


\section{LA PENSÉE NARCISSIQUE}

Nous pouvons accomplir un pas de plus en réfléchissant maintenant sur un extrait de Blondel (1934):

... la pensée comporte trois significations qu'on ne peut ni isoler, ni réduire à l'unité, ni simplement juxtaposer. Tour à tour, elle est ce qui est pensé ou tout au moins pensable, - elle est ce qui est pensant, produisant, agissant, - elle est ce rapport mystérieux, entre ce qui semble les deux données précédentes; (Blondel, 1934, p. 6) ${ }^{3}$

Il faut accorder à Blondel qu'un objet qui serait à la fois (ou tour à tour?) ce qui est pensé, ce qui est pensant et la relation entre les deux, serait en effet quelque chose de bien spécial. Il devrait s'agir d'une fonction binaire - appelons-la " $\mathrm{R}$ " - qui appartiendrait à son propre domaine aussi bien qu'à son co-domaine, rendant ainsi possible un énoncé de forme « $R$ a la relation $R$ avec $R$ » ( $R R R »)$. On sait que la théorie des types logiques, même dans ses versions les plus accomodantes, considère de tels énoncés comme dénués de sens. Mais, quoi qu'il en soit de la théorie des types, il est facile de voir qu'on peut très bien parler de pensée sans faire aussitôt lever le spectre d'une relation aussi acrobatique et aussi narcissique. Dans le fait exprimé par «Titus pense à Bérénice», il n'y a de toute évidence aucun être, ni aucune fonction qui soit à la fois sujet, objet et relation entre les deux; il doit notamment être possible de penser à autre chose qu'à soi-même.

Je suggère que la meilleure façon de donner sens à la phrase de Blondel serait de l'interpréter comme la reconnaissance de l'équivocité du mot «pensée», qui en effet dénote tantôt l'objet pensé, tantôt la relation "penser à» ou "penser que», et tantôt encore - mais très rarement - l'objet pensant ${ }^{4}$.

Résumons-nous: le principe d'intentionnalité pouvait apparaître comme un principe ontologique ou transcendantal d'une extrême importance; il se révèle n'être qu' un postulat de signification, un principe analytique trivialement vrai. La découverte par

3. Cité par Renault (1979), p. 8. J'utilise cette citation prise hors-contexte parce qu'elle est en elle-même intéressante pour mon propos. On comprendra que je n'entends nullement m'engager de la sorte dans un débat sur l'interprétation de Blondel.

4. Cette dernière acception du mot "pensée", pris comme dénotant l'objet pensant, se retrouve principalement chez certains philosophes spiritualistes marqués par la lecture de Descartes. Dans l'usage ordinaire, il ne suit pas de «Pierre pense à quelque chose» que Pierre soit lui-même une pensée. 
Blondel d'une mystérieuse fonction tricéphale semblait prouver la nécessité d'un type ontologique spécial, capable à lui seul de réfuter toutes les théories des types d'inspiration russellienne; elle se révèle n'être que la reconnaissance d'une équivocité verbale.

Au moment de s'engouffrer dans un débat sur l'intentionnalité, il y a là de quoi nous mettre la puce à l'oreille. Les fantômes ontologiques ou phénoménologiques qui hantent ce gouffre ne seraient-ils que les ombres amplifiées de certains phénomènes de surface très ordinaires, à savoir des phénomènes linguistiques?

L'ÉTRANGE LOGIQUE DU VERBE «AIMER»

Une approche logico-sémantique des énigmes de l'intentionnalité requerrait une analyse minutieuse des propriétés syntaxiques et sémantiques des énoncés qui décrivent des états intentionnels. Il y a tout à parier qu'une fois cette analyse menée à bien, les énigmes en question seraient sur le plan intellectuel entièrement résolues, même si d'ailleurs ce type de solution devait, ce qui est probable, nous laisser plutôt insatisfaits sur le plan émotif. Pour l'instant cependant, il convient de reconnaître, pour éviter toute mystification, que la transposition logico-sémantique ne permet pas encore une solution définitive, même sur le plan intellectuel; on verra, à y regarder de plus près, que les particularités sémantiques des énoncés décrivant des états intentionnels se laissent ramener à celles des énoncés dits «intensionnels» (avec un «s»), ces énoncés à l'intérieur desquels il n'est pas toujours possible de remplacer une expression par une autre ayant même dénotation sans changer la valeur de vérité de l'énoncé lui-même. Or, on sait que l'analyse sémantique de tels énoncés se heurte encore aujourd'hui à de sérieuses difficultés techniques. Pour le moment donc, mon hypothèse de travail n'affirme rien de plus que ceci:

1) les énigmes de l'intentionnalité - du genre de celles qui ont été brièvement formulées dans le premier paragraphe de cet article - se ramènent aux énigmes de l'intensionnalité (avec un «s»);

2) ainsi reformulées, elles ne sont pas encore résolues pour autant, mais elles sont en principe plus facilement solubles.

Considérons, pour rendre tout cela tout intuitif, un énoncé typique décrivant un état intentionnel comme: 


\section{- Titus aime Bérénice}

Et notons en premier lieu que, dans certains usages, cet énoncé présente la même forme logique que n'importe quel énoncé relationnel ordinaire, comme "Adam est le père de Caïn", la forme $a R b$, où « $R$ » est un prédicat relationnel binaire, et «a» et «b» sont des constantes individuelles. Ce genre d'usage ne suscite donc aucune difficulté spéciale pour l'analyse sémantique ${ }^{5}$ les noms propres "Titus" et "Bérénice» dénotent certains individus empiriquement identifiables, et le verbe «aimer» indique l'existence d'une certaine relation, au moins indirectement attestable, entre les deux individus en question. Certes, la vérification empirique d'un énoncé de forme "A aime $B$ " rencontre certains obstacles, mais il n'en va pas autrement, cela est bien connu, pour la vérification de " $A$ est le père de $B$ ". L'important est qu'il est possible, dans cet usage, de substituer à chacun des deux noms propres n'importe quelle expression référentielle ayant la même dénotation sans changer la valeur de vérité de l'énoncé. Ainsi, puisque l'expression «la fille d'Agrippa $\mathrm{I}^{\mathrm{er}}$ » dénote le même individu que le nom «Bérénice», on peut admettre, toujours selon l'usage particulier que nous examinons ici, que l'énoncé «Titus aime la fille d'Agrippa $\mathrm{I}^{\mathrm{er}}{ }_{\text {}}$ doit toujours avoir la même valeur de vérité que «Titus aime Bérénice», même si Titus pour sa part ignore absolument que Bérénice soit en fait la fille d'Agrippa $\mathrm{I}^{\mathrm{er}}$. Nul besoin ici d'invoquer l'existence d'entités intrinsèquement relationnelles, ni d'objets noématiques différents des objets réels.

Les inquiétudes de l'approche logico-sémantique commencent quand on remarque l'existence d'une autre interprétation ou d'un autre usage - de «Titus aime Bérénice», selon laquelle le nom «Bérénice» n'est pas pris de façon purement référentielle, ou, pour parler comme Quine (1943), de façon désignative. À supposer que Titus existe, il pourrait même être vrai, dans un certain usage au moins, qu'il aime Bérénice, sans que cela n'implique l'existence réelle de Bérénice: Titus, par exemple, aurait pu être abusé par des récits inventés de toutes pièces et développer

5. Je ne dis pas que l'analyse sémantique ne rencontre ici aucun problème. Je dis qu'elle ne rencontre aucun problème spécial. De toute évidence, l'analyse d'un énoncé de forme aRb requiert au minimum une sémantique des noms propres, ce qui déjà suscite bien des difficultés. Mais elles sont inévitables de toute façon. 
une passion folle pour l'inexistante hérö̈ne de ces histoires. On retrouve ici, formulé en termes sémantiques, le vieux problème du statut des objets non-existants: si Bérénice n'existe pas, et si pourtant il est vrai que Titus aime Bérénice, quel est, dans cette dernière phrase, l’objet dénoté par le nom «Bérénice»? Quelle que soit la réponse, la seule possibilité de soulever la question indique déjà une différence notable entre la logique d'un verbe d'état intentionnel comme «aimer», pris selon l'usage que nous considérons maintenant, et celle d'un prédicat relationnel ordinaire comme «être père de»: Adam, lui, ne saurait être le père de Caïn si Cain n'existe pas dans le même univers de discours.

La propriété sémantique la plus remarquable du verbe «aimer» selon ce nouvel usage est que le nom qui figure comme complément d'objet ne peut plus dans tous les cas être remplacé dans l'énoncé par une autre expression ayant ordinairement même dénotation sans que la valeur de vérité de l'énoncé n'en soit affectée. Il serait de la sorte facile, dans une humeur quinienne, de construire avec le verbe "aimer" des paradoxes apparents. Soit par exemple le paradoxe suivant, que j'appellerai «le paradoxe du chien fidèle» et que je dédie tout particulièrement à ceux qui disent vouloir être aimés pour eux-mêmes:

Le chien d'Albert, Fido, comme la plupart des chiens, aime son maître. Par conséquent: - "Fido aime Albert" est vrai. Cependant, il arrive quelquefois qu'à l'insu de Fido, Albert, qui, lui, n'aime pas son chien, se déguise, maquillant par des procédés fort sophistiqués jusqu'à son odeur. Il coiffe alors un chapeau rouge, s'arme d'un bâton et persécute la pauvre bête. Inutile de dire que Fido, qui ne reconnaît pas son 'maître, déteste l'homme au chapeau rouge. Il n'y a ici aucune ambivalence émotive: il serait carrément faux d'affirmer que Fido aime l'homme au chapeau rouge. Mais comme l'homme au chapeau rouge et Albert ne font qu'un, on peut conclure par substitution des identiques: — «Fido aime Albert» est faux.

Nous avons ainsi engendré une contradiction, au moins apparente ${ }^{6}$.

6. Ce paradoxe ne peut pas être résolu, comme on le croira peut-être à première vue, par une indexation temporelle des propositions en cause. "Aimer» étant un terme dispositionnel, "Fido aime son maître» devrait être vrai même pour les moments où Fido doit affronter l'homme au chapeau rouge; et corrélativement, "Fido aime l'homme au chapeau rouge» doit être faux pour les moments où Fido dort en toute quiétude aux pieds de son maître. 
De telles spéculations, que les phénoménologues appelleraient peut-être des variations imaginaires, permettent de constater que, dans certains de ses usages au moins, le prédicat «aimer» ne se comporte pas prima facie comme un prédicat extensionnel binaire du genre «être père de». Caïn aurait beau mettre un chapeau rouge, ou même se dissimuler dans une tombe, cela ne changerait rien à la valeur de vérité de «Adam est le père de Caïn .

Le même phénomène se retrouve, cela est bien connu, dans le cas d'énoncés comme "Noé pense qu'il pleut», qui décrivent ce qu'il est convenu d'appeler, depuis Russell (1940), des «attitudes propositionnelles», c'est-à-dire les attitudes intentionnelles de certains individus face à des contenus propositionnels. Les foncteurs "pense que ...», «croit que ...», «souhaite que ...», etc., engendrent, pour parler comme Quine (1960), des contextes référentiellement opaques, c'est-à-dire des contextes linguistiques à l'intérieur desquels la substitution des identiques n'est pas toujours possible: «Edouard croit que l'auteur de Waverley est Scott» n'est pas logiquement équivalent à «Edouard croit que Scott est Scott», bien que Scott soit en fait identique à l'auteur de Waverley.

\section{APPROCHES DE L'INTENSIONNALITÉ (AVEC UN «S»)}

Il ressort des considérations précédentes que les énigmes de l'intentionnalité (avec un "t»), lorsqu'elles sont abordées par le biais de l'analyse logico-linguistique, se ramènent pour une large part aux énigmes de l'intensionnalité (avec un "s»). Un langage en effet est dit «intensionnel" s'il ne permet pas toujours la substitution des identiques salva veritate. Les contextes linguistiques particuliers dans lesquels cette substitution n'est pas admise sont appelés "contextes intensionnels», et les foncteurs qui les engendrent (comme «aimer» ou «croire que», dans les exemples ci-dessus) sont en conséquence des foncteurs intensionnels. Reformulée en termes sémantiques, l'énigme centrale devient ainsi la suivante: comment peut-on rendre compte du comportement logique particulier des foncteurs intensionnels?

Certes, ce déplacement de l'intentionnalité comme phénomène de subjectivité (transcendantale ou empirique) à l'intension- 
nalité comme phénomène linguistique ne règle pas ipso facto tous les problèmes, loin de là. On sait que, depuis Frege, la logique et la sémantique contemporaines butent avec régularité contre les obstacles que les contextes intensionnels opposent à une théorisation et une formalisation rigoureuses. Mais le gain n'est pas annulé pour autant. Quel que soit le traitement réservé à l'intentionnalité avec un «t», les problèmes de l'intensionnalité avec un «S» continueront tout de même de requérir l'attention: le déplacement proposé ici présente donc au moins l'avantage de réduire le nombre d'énigmes théoriques auxquelles les philosophes sont confrontés. Nous gagnons également en généralité puisque les foncteurs intensionnels constituent une classe beaucoup plus large que celle des seules expressions renvoyant à des états intentionnels: cette classe contient aussi tous les autres foncteurs modaux, comme «il est nécessaire que...», «il est possible que ...», «il est interdit que....» etc.

En outre, nous disposons déjà, pour l'analyse des langages intensionnels, d'appareils conceptuels extrêmement précis et sophistiqués, susceptibles sur le plan formel de combler les voeux de Husserl lui-même en faveur du développement de la philosophie comme science rigoureuse; c'est tout le domaine de la logique modale qui peut être mis ici à contribution. Nous avons, enfin, une idée relativement claire des voies qui s'offrent à nous pour tenter de résoudre, par l'analyse sémantique, les énigmes de l'intensionnalité. Je voudrais brièvement rappeler les principales d'entre elles.

1) On peut tenter de traduire les langages intensionnels dans des langages extensionnels - où la substitution des identiques est toujours possible salva veritate - en proposant une analyse des foncteurs intensionnels en termes relationnels ordinaires. "Croire que» par exemple serait, dans certains cas du moins, remplacé par un prédicat relationnel unissant des individus à des phrases. Dire qu'Edouard croit que l'auteur de $W$ averley est Scott reviendrait à poser une relation d'un type déterminé entre l'individu Édouard et une certaine phrase d'une langue donnée connue de Édouard, par exemple la phrase anglaise «the autbor of $W$ averley is Scott". Si par convention nous posons que "WS" est, dans notre nouveau langage, le nom de cette phrase anglaise (et non pas sa traduction), l'énoncé intensionnel original "Édouard croit que 
I'auteur de Waverley est Scott» sera traduit dans un nouvel énoncé entièrement extensionnel de forme «Édouard a telle relation avec WS» (par exemple: «Édouard est disposé à affirmer WS») dans lequel "WS» peut être remplacé salva veritate par toute autre expression dénotant aussi la même phrase anglaise. Il n'y a plus dans ce cas aucun problème à constater que "WS" ne peut pas être remplacé salva veritate par une autre expression «SS" dénotant par exemple la phrase «Scott is $S$ cott» puisque dès lors il est clair que «WS» et «SS" dénotent deux objets différents (même si ces deux objets sont des phrases synonymes entre elles) ${ }^{7}$.

De même, «Titus aime Bérénice» pourrait être traduit par quelque chose comme "Titus est disposé à ressentir telles émotions $E$ lorsqu'il est placé dans telles circonstances $C »$. Les émotions seraient alors conçues comme des propriétés nonrelationnelles et la nouvelle formulation, prise dans son ensemble, affirmerait l'existence d'une relation ternaire entre Titus, les émotions $\mathrm{E}$ et les circonstances $\mathrm{C}$. Elle serait en principe extensionnelle.

2) On peut, dans la foulée de Frege et de Church, essayer de reconstruire les états intentionnels comme des relations entre des individus (par exemple des humains) et certaines entités intensionnelles non-linguistiques et non-psychologiques comme des sens, des concepts ou des propositions, qui ne sont pas de même type logique que les individus de l'univers de discours ${ }^{8}$ et qui correspondent grosso modo aux noèmes de la phénoménologie husserlienne. Dire que Noé croit qu'il pleut serait poser une telle relation entre Noé et une certaine proposition, l'entité objective abstraite qui est le sens des phrases «il pleut», «it is raining», etc. Cette solution à caractère platonicien est plus simple à manipuler que la précédente mais elle tend à complexifier considérablement l'ontologie en admettant l'existence objective des entités abstraites, ce qui est plutôt déroutant pour l'intuition.

À propos de ces deux méthodes, Carnap (1963) déclare:

C'est seulement après que des recherches plus poussées auront été

7. Une solution de ce genre est esquissée dans Carnap (1956), sect. 13-15.

8. Pour de brefs plaidoyers en faveur du recours à de telles entités intensionnelles, voir Frege (1918), ou encore Church (1956). On peut également penser au fameux troisième monde de Popper (voir Popper, 1972, ch. 3). 
poursuivies que nous serons en mesure de déterminer laquelle des deux méthodes présente les avantages les plus grands (p. 898, trad. par moi).

Depuis 1963, les développements de la logique intensionnelle ont permis d'approfondir surtout la deuxième méthode, bien que de nombreux philosophes demeurent encore fort sympathiques à l'extensionnalisme ${ }^{9}$. Pour autant que je puisse voir, les résultats ne sont pas encore décisifs.

3) Une autre approche, prometteuse mais très peu explorée, est brièvement suggérée par Quine (1960) à propos des attitudes propositionnelles, et peut-être est-elle général isable à l'analyse de tous les foncteurs intensionnels. Elle consisterait essentiellement à ne plus les traiter du tout comme des prédicats relationnels. Selon cette analyse par exemple, le «p» de "A croit que p» ne serait pas conçu comme le terme d'une relation et on n'aurait donc plus à se demander de quelle sorte d'objet il peut bien s'agir. Le verbe «croire que» deviendrait une expression, elle-même dépourvue de dénotation, qui, appliquée à une phrase «p», permet la construction d'un prédicat unaire complexe «croire-que-p», dont «p» serait un constituant immédiat ${ }^{10}$.

Cette solution est attrayante parce qu'elle évite la délicate question du statut des objets intensionnels avec un «s» (et donc aussi des objets intentionnels avec un "t»). Reste à savoir si elle peut être rendue opératoire (ce que Quine n'a pas fait) et surtout si elle ne se ramène pas tout simplement à la première méthode évoquée plus haut. Quoi qu'il en soit, mon propos n'est pas ici de résoudre ces problèmes, mais de montrer comment certaines énigmes relatives à l'intentionnalité (notamment la question du statut des objets intentionnels ou noèmes) peuvent être abordées dans la perspective de la sémantique formelle contemporaine.

\section{QUI SONT LES SUJETS INTENTIONNELS?}

On remarquera que jusqu'ici les difficultés n'ont concerné que le verbe et le complément d'objet des phrases qui renvoient à des états intentionnels. Beaucoup de philosophes, cependant, semblent croire que c'est plutôt le sujet de ces phrases qui fait problème: c'est là, à leurs yeux, la fameuse question du statut de

9. Voir par exemple Gochet (1972).

10. Voir Quine (1960), pp. 215-16. 
la subjectivité. Or, l'analyse sémantique porte à conclure, dans un premier temps du moins, qu'un mot comme "Noé» ne se comporte pas de façon différente dans «Noé pense qu'il pleut» et dans «Noé a la barbe longue»: il s'agit tout simplement dans les deux cas d'un nom pris référentiellement (ou de façon désignative), tout comme «Montréal» dans «Montréal est une grande ville». Le caractère intensionnel du foncteur «pense que» dans «Noé pense qu'il pleut» ne suscite sur le plan sémantique aucune énigme particulière quant à l'interprétation de son sujet. À supposer par exemple que Noé existe au moment présent dans l'univers de discours, les difficultés spéciales posées par la vérification empirique de «Noé pense qu'il pleut» tiennent toutes à l'interprétation de "pense que» et non pas à celle de «Noé», ce dernier mot pouvant facilement être compris comme le nom propre d'un certain organisme biologique empiriquement identifiable.

Il faut cependant aller plus loin: bien que "Noé», lorsqu'il est pris comme sujet d'un verbe intensionnel, continue de se comporter sur le plan sémantique de la même façon qu'un nom propre ordinaire, il reste néanmoins que, dans le discours quotidien, les verbes qui dénotent des états intentionnels n'admettent pas n'importe quel nom comme sujet: «la couleur rouge pense qu'il pleut" apparaît comme un énoncé mal formé. Peut-être voudra-t-on en conclure que, pour que les phrases formées à l'aide d'un verbe comme "pense que" soient sémantiquement interprétables, leurs sujets doivent dénoter des individus bien spéciaux dotés de ces propriétés particulièrement énigmatiques que l'on résume habituellement sous l'étiquette «intentionnalité». Nous serions alors presque ramenés à notre point de départ: la reconnaissance, parmi les individus de l'univers de discours, d'une variété ontologique irréductible, celle des sujets intentionnels.

Mais n'allons pas trop vite. Si la phrase «la couleur rouge pense qu'il pleut» n'est pas simplement fausse, mais sémantiquement ininterprétable, c'est-que nous avons ici affaire non pas à une difficulté empirique, mais à une incongruité logique, du même genre que les infractions commises par un énoncé comme «d'incolores idées vertes dorment furieusement». L'inacceptabilité sémantique de telles phrases ne tient pas aux propriétés empiriques des objets réels, mais aux règles des langages dans 
lesquels elles sont formulées. Il s'agit ici de ce que Carnap (1928) appelle une "confusion des sphères»" ${ }^{11}$, et Ryle (1949) un "category-mistake» ${ }^{12}$ : ce sont là des erreurs linguistiques.

Quoi qu'il en soit, il faut reconnaitre que les foncteurs qui renvoient à des états intentionnels imposent certaines contraintes particulières relativement aux termes qu'ils peuvent logiquement accepter comme sujets, et c'est là un problème que toute approche logico-sémantique de ces foncteurs se doit d'examiner en détail. Remarquons seulement pour l'instant - mais remarquons-le avec insistance - que, dans le langage ordinaire, l'ensemble des noms qui sont logiquement admissibles à titre de sujets des verbes d'états intentionnels comme «pense que» est beaucoup plus large que l'ensemble des noms d'individus humains: même formulée en termes phénoménologiques, la problématique de l'intentionnalité ne concerne pas spécifiquement la sphère de la subjectivité humaine individuelle.

Dans l'usage ordinaire - il me semble que les philosophes l'oublient trop souvent - il est parfaitement loisible de produire des énoncés comme les suivants:

- le chien Fido pense que son maitre est sur le point d'arriver

- le gouvernement américain souhaite que les otages lui soient rendus ${ }^{13}$

- les tournesols aiment le soleil

- la foule pense que l'arbitre favorise l'adversaire

- la distributrice à cigarettes refuse de rendre la monnaie

- la société québécoise est consciente de sa spécificité

Ceux qui ont déjà joué aux échecs contre un ordinateur savent que l'on peut tout naturellement parler de cet adversaire mécanique avec les mêmes verbes d'états intentionnels que l'on appliquerait spontanément à un joueur humain: il pense que ...., il craint que..., il calcule que ..., il veut que ... etc.

Si quelqu'un croit que tous ces usages sont dérivés ou métaphoriques par rapport à celui qui attribue des états intention-

11. Voir Carnap (1928), sections 30 et 31 .

12. Voir Ryle (1949), p. 17 et ss.

13. Notons que, dans une certaine interprétation au moins, cet énoncé pourrait être vrai même si, individuellement et en son for intérieur, chaque membre du gouvernement souhaitait le contraire. 
nels à des personnes humaines, il devrait tenter d'en fournir une démonstration. Dans la conversation quotidienne en tout cas, les animaux, les entités administratives, les machines, les collectivités se présentent comme des candidats aussi «naturels» que les êtres humains au titre de sujets intentionnels. Cela porte à croire que la question de l'intentionnalité a été, dans certaines discussions philosophiques, trop étroitement associée au problème du statut des états mentaux et de la subjectivité humaine ${ }^{14}$. Sans doute est-ce que Husserl lui-même voulait éviter en proposant de l'intentionnalité une approche transcendantale plutôt que psychologique et en mettant l'être humain empirique à distance de ce qu'il appelle la conscience pure ${ }^{15}$.

Mais, ainsi dépsychologisée, la conscience pure n'est sans doute rien d'autre que la logique d'un certain langage. S'il en est ainsi, parler de structures intentionnelles, c'est s'exprimer en mode matériel (au sens de Carnap, 1934) pour traiter de certaines structures logico-linguistiques particulières qui engendrent des contextes intensionnels (avec un «s»).

\section{LES ÊTRES INTENTIONNELS EXISTENT-ILS?}

Quant à savoir s'il y a bien dans le monde des êtres réels tels qu'on ne puisse en parler adéquatement sans recourir, à un moment ou l'autre, à des foncteurs intensionnels, c'est là une pseudo-question, si du moins l'on accepte la distinction proposée par Carnap (1950) entre deux sortes de questions relatives à l'existence ou à la réalité de certaines entités.

Il appelle les premières des «questions internes». Elles présupposent l'acceptation d'un certain cadre linguistique («linguistic framework») et elles invitent à appliquer les règles particulières inhérentes à ce cadre pour déterminer l'acceptabilité et la valeur de vérité d'un énoncé formé à l'aide du quantificateur existentiel (ou l'équivalent). Voici quelques exemples familiers de questions internes:

- existe-t-il une ville plus grande que New York?

- existe-t-il un nombre entier plus grand que trois et plus petit que cinq?

14. Faur-i] souligner que ces considérations n'impliquent rien de tel qu'une négation de la spécificité des êtres humains par rapport aux machines ou aux animaux?

15. Voir notamment Husserl (1913), section II, chap. III. 
- existe-t-il une couleur plus brillante que le rouge?

- existe-t-il une société qui pense que ses voisins lui sont supérieurs?

À ces questions internes, Carnap oppose les questions externes, qui portent sur l'existence ou la réalité du domaine total d'objets dans lequel prennent leurs valeurs les variables permises par le cadre linguistique, par exemple:

- les objets matériels existent-ils?

- les nombres existent-ils?

- les universaux existent-ils?

- le monde existe-t-il?

Par opposition à une question de forme "existe-il quelqu'un qui pense ceci ou cela?», la question «les êtres intentionnels existentils?" appartient à cette seconde catégorie.

De telles interrogations, la plupart du temps suscitées par des philosophes, mettent en question les règles les plus fondamentales d'un langage, celles qui concernent la façon dont, préalablement à la position de tout énoncé particulier formé dans ce langage, il organise son propre univers de discours. On ne saurait par conséquent y répondre dans le langage même qui est ainsi mis en question sans tomber dans la trivialité et sans passer complètement à côté de la question. Les nombres existent-ils? On pourrait bêtement répondre: bien sûr que oui, puisqu'il existe un nombre plus grand que trois et plus petit que cinq, et ainsi de suite pour chaque nombre! De même: existe-t-il des êtres intentionnels? Oui, puisque Titus aime Bérénice et Fido aime Albert! De telles réponses ne sont évidemment pas pertinentes, et la raison pour laquelle elles ne sont pas pertinentes est que justement chacune présuppose le cadre linguistique mis en cause par la question correspondante.

Entreprendra-t-on d'y répondre dans un autre langage qu'aussitôt des difficultés analogues surgiront. Ou bien le langage en question admet le domaine d'entités qui fait problème, ou bien il ne l'admet pas. Dans un cas comme dans l'autre, la réponse à notre question externe, qui semblait porter sur la réalité la plus intime des choses, découlera directement des règles mêmes du langage dans lequel nous tenterons d'y répondre. C'est dire qu'à tout coup la réponse sera analytique et ratera par 
conséquent les préoccupations métaphysiques de celui qui posait la question.

Il n'y a, suggère Carnap, qu'une seule façon féconde de traiter de telles questions. C'est de les recevoir comme des questions pratiques, et non pas théoriques, concernant la pertinence relativement à certains objectifs d'adopter ou de ne pas adopter tel ou tel langage et l'univers de discours qui l'accompagne automatiquement. Les questions externes seraient ainsi ramenées à des préoccupations pratiques relatives au choix d'un appareil linguistique: adopterons-nous, pour telles ou telles fins, le langage de l'arithmétique, ou celui des probabilités, ou un système intensionnel quelconque, ou encore le français, l'anglais, l'esperanto ou le fortran? Selon cette interprétation, une question comme "les propriétés existent-elles?» devient quelque chose comme: déciderons-nous d'adopter (pour telles ou telles fins qui resteraient à spécifier) un langage dans lequel il est possible de quantifier sur les prédicats du premier ordre pour construire des énoncés de forme «il existe un $F$ tel que Fa»?

De même, la question «les êtres intentionnels existent-ils» deviendrait: déciderons-nous d'adopter (pour telles ou telles fins qui resteraient à spécifier) un langage comportant certains foncteurs intensionnels comme «pense que»? Ce n'est pas simplement en scrutant le monde, fût-ce avec le regard perçant du métaphysicien, que nous pourrons répondre à de telles questions, puisqu'elles requièrent à la fois l'identification de certains objectifs et le recours à une échelle critériologique pour l'évaluation des moyens à prendre ${ }^{16}$.

\section{BILAN PROVISOIRE}

Si maintenant nous faisons le point sur l'ensemble de notre démarche, nous voyons que la reformulation des énigmes de l'intentionnalité dans les termes de l'approche logico-sémantique nous conduit aux résultats suivants:

16. Ceux que préoccupent les rapports de la théorie et de la pratique noteront ici que cette interprétation des questions externes comme portant sur le choix d'un langage permet d'accepter comme pertinentes pour leur discussion de nombreuses considérations d'ordre axiologique, et en particulier des considérations morales et politiques. 
1) Le problème de penser les êtres intrinsèquement relationnels dont le principe d'intentionnalité suggère l'existence (les sujets intentionnels) apparaît comme un faux problème, puisque, quelle que soit notre interprétation finale des foncteurs renvoyant à des états intentionnels, leurs sujets, eux, peuvent toujours être interprétés de façon purement référentielle comme dénotant certains individus de l'univers de discours, et notamment des individus empiriquement reconnaissables, comme des êtres humains.

2) Les foncteurs qui renvoient à des états intentionnels imposent néanmoins certaines restrictions sévères sur la classe des termes qu'ils admettent comme sujet. L'analyse logico-sémantique devrait être en mesure de caractériser de façon précise les dites restrictions, mais à ma connaissance cela n'a pas encore été fait de façon satisfaisante. Dire par exemple que cette classe est la classe des êtres intentionnels est purement tautologique et sans grand intérêt. Dire que c'est la classe des êtres humains est certainement faux, du moins pour les langues naturelles qui me sont familières (comme le français et l'anglais). Il y a donc là un problème ouvert.

3) Quant à savoir s'il convient, pour parler de telles ou telles catégories d'objets, de recourir à des foncteurs intensionnels, ça n'est pas là une question empirique, mais plutôt matière à décision. Ce qui nous reconduit à l'un des problèmes philosophiques les plus importants de notre époque (malgré son allure assez abstraite): quelle serait la forme logique exacte d'un argument permettant de conclure que tel langage est plus approprié que tel autre pour l'atteinte de tels objectifs déterminés?

4) Le problème du statut des objets noématiques se ramène à celui des objets intensionnels (avec un "s"). Il serait présomptueux d'affirmer que ce problème est à l'heure actuelle définitivement réglé. Mais nous voyons du moins assez clairement les voies qui s'offrent à nous. Quelle que soit la solution qu'on adopte, la question du statut, relationnel ou non, des états intentionnels sera tranchée du même coup. 
À mon sens, ce sont là des résultats appréciables. Le déplacement du point de vue phénoménologique au point de vue logicosémantique, s'il ne règle pas d'un seul coup toutes les difficultés philosophiques, permet néanmoins de repérer, avec plus de netteté, les questions qui restent ouvertes, et suggère par là même des façons relativernent précises de les aborder. Mais, bien sûr, tout cela n'est encore que suggestion. Une démonstration précise devrait s'appuyer sur une comparaison beaucoup plus systématique des deux approches en question, relativement à certains problèmes particuliers clairement identifiés. Peut-être serionsnous alors plus en mesure de jauger l'intérêt propre de chacune des deux méthodes, de reconnaitre les domaines dans lesquels chacune est la plus féconde et d'apercevoir, sous leurs divergences manifestes, certaines affinités fondamentales.

Département de philosophie

Université du Québec à Trois-Rivières 


\section{BIBLIOGRAPHIE}

BLONDEL, M., 1934. La Pensée. Paris, P.U.F.; 2 vols.

CARNAP, R., 1928. Der Logische Aufbau der Welt; Trad. angl.: The Logical Structure of the World. Berkeley/Los Angeles, University of California Press, 1969.

CARNAP, R., 1934. Logische Syntax der Sprache. Trad. angl.: The Logical Syntax of Language, London, Routledge and Kegan Paul, 1937.

CARNAP, R., 1950. «Empiricism, Semantics and Ontology». Revue Internationale de Philosophie, 4, 1950, pp. 20-40; repris dans Carnap, 1956, pp. 205-21.

CARNAP, R., 1952. "Meaning Postulates». Philosophical Studies, 3, pp. 65-73; repris dans Carnap, 1956, pp. 222-29.

CARNAP, R., 1956. Meaning and Necessity. 2e éd. revue, Chicago/London, The University of Chicago Press.

CARNAP, R., 1963. «Replies and Systematic Expositions», in P.A. SchilpP (éd.), The Philosophy of Rudolf Carnap. La Salle, Ill.: Open Court, et London: Cambridge University Press, pp. 859-1013.

CHURCH, A., 1956. "Propositions and Sentences", in A. Church, N. Goodman et I.M. Bochenski, The Problem of Universals. A Symposium. Notre Dame, Ind.: University of Notre Dame Press, pp. 1-11.

FREGE, G., 1918. «Der Gedanke». Beiträge zur Philosophie des deutschen Idealismus, 1, pp. 58-77; Trad. française in G. Frege, Écrits logiques et philosophiques. Paris: Seuil, 1971, pp. 170-95.

GOCHET, P., 1972. Esquisse d'une théorie nominaliste de la proposition. Paris: Armand Colin.

HUSSERL, E., 1913. Ideen zu Einer Reinen Phaenomenologie und Phaenomenologischen Pbilosopbie. Vol. I; Trad. franç.: Idées directrices pour une phénoménologie, Paris: Gallimard, 1950.

LINSKY, L., (éd.) 1952. Semantics and the Philosopby of Language. Urbana: University of Illinois Press.

LYOTARD, J.-F., 1961. La phénoménologie, Paris: P.U.F. (Que sais-je? no 625).

POPPER, K., 1972. Objective Knowledge. Oxford: Oxford Univ. Press.

QUINE, W.V.O., 1943. "Notes on Existence and Necessity». The Journal of Philosophy, 40; Repris dans Linsky (1952) pp. 75-91.

QUINE, W.V.O., 1960. Word and Object. Cambridge, Mass.: The M.I.T. Press.

RENAULT, M., 1979. Le Singulier. Montréal: Bellarmin, et Paris/Tournai: Desclée.

RUSSELL, B., 1940. An Inquiry into Meaning and Truth. London: Allen and Unwin.

RYLE, G., 1949. The Concept of Mind. London: Hutchinson (rééd. Penguin University Books, 1973). 